\title{
Una inscripción funeraria del monasterio de San Esteban de Bañolas (siglo XI)
}

\author{
Javier de Santiago Fernández *
}

\begin{abstract}
RESUMEN
ABSTRACT

En el presente artículo se estudia una

The present article studies inscripción procedente del monasterio de San Estaban de Bañolas (Gerona), datada en el año 1003 d.C. El método

seguido para el análisis del citado epigrafe ha tenido en cuenta tanto los elementos externos: materia, forma $y$ escritura, como los internos: lengua y pensamiento. Tales elementos han sido analizados en conexión con su contexto histórico y epigráfico, por cuanto la presente inscripción representa un magnífico ejemplo de las características del grupo epigráfico catalán, en el que se reflejan perfectamente las caracteristicas procedentes del mundo franco y que, al mismo tiempo, lo separan del panorama imperante en el resto de la Península. an inscription from San Esteban de Bañolas monastery (Gerona). It is dated in 1003 B.C. The method I have used is based on the study of external (material, form and writing) and internal elements (languaje and thought). These elements are analysed with its historic and epigraphic context. This inscriptions is a very good example of epigraphic group of Catalonia. This group is influenced by Frankish world and it is no like other epigraphic groups in Spain. So we can make out that an inscription, and the epigraphic production in general, reflects its society.

De este modo es posible apreciar como una inscripción concreta y la producción epigráfica en general es resultado y reflejo de una sociedad concreta.
\end{abstract}

* Prof. Titular de Epigrafía y Numismática. Universidad Complutense de Madrid. 
Quiero comenzar este trabajo mostrando mi agradecimiento a Doña María Rosa Ferrer i Dalgà, del Museu Diocesà de Girona, por facilitarme el conocimiento de esta inscripción procedente del monasterio de San Esteban de Bañolas, además de la fotografía y los datos técnicos que me han permitido realizar el estudio. Igualmente por su amabilidad y disposición al atender todas mis solicitudes al respecto.

La inscripción procede del monasterio de San Esteban de Bañolas (Gerona). Fue hallada en 1969 en las proximidades de la jamba de la izquierda del arco triunfal del presbiterio moderno, como consecuencia de las tareas de excavación arqueológica que allí se emprendieron aprovechando las obras de acondicionamiento del templo para adaptarlo a las nuevas necesidades litúrgicas derivadas del Concilio Vaticano II $^{1}$. Esas excavaciones sacaron a la luz bastantes sepulturas halladas en las proximidades de la inscripción. El hallazgo se produjo aproximadamente a un metro del primitivo pavimento, realizado en roca natural, del templo primitivo, construido totalmente en piedra y consagrado en el 957 por Arnulfo, obispo de Gerona. La inscripción posteriormente se situó en el claustro, de donde fue retirada con ocasión de las obras de restauración del monasterio.

Su interés, como el de tantos documentos epigráficos de época medieval, está en ser un testimonio fiel de la vida de un monasterio, de formar un trozo, aunque sea pequeño, de su historia y de las personas que la fueron construyendo, así como en constituir una aportación más para el conocimiento de la epigrafía medieval. La mayor parte de las inscripciones medievales constituyen parte de la vida de la Iglesia medieval, pues no en vano la epigrafía medieval es de origen fundamentalmente eclesiástico. En la mayor parte de las ocasiones son los clérigos los que escriben y son los monasterios e instituciones eclesiásticas quienes utilizan los textos epigráficos como medio de comunicación a través del cual ofrecen información sobre la liturgia, sobre sus santos y mártires, sobre sus personajes ilustres y, en fin, sobre todo aquello que pueda tener interés en un momento concreto. La producción de inscripciones era una más de las actividades que se realizaron en algunos monasterios medievales, en directa relación con el scriptorium o centro productor de códices, labor tradicionalmente mucho más valorada por los investigadores.

El monasterio de San Esteban se encuentra en un extremo de la villa de Bañolas, en la provincia de Gerona. Las primeras noticias que tenemos

La noticia de dicho hallazgo y un inicial estudio de la inscripción en J. Marques Casanovas, "Descubrimiento de un epitafio del año 1003 en el templo monasterial de San Esteban, de Bañolas", Revista de Gerona, 59 (1972), pp. 23-27. Ver también A. Pladevall (dir.), Catalunya Romànica, vol. XXVI, Barcelona, 1997. 
de su constitución datan de los años iniciales del siglo Ix. Fue fundado en torno al 812 por el monje Bonito, al parecer de origen aquitano, con la autorización del conde Odiló. En el año 822 ya se menciona la regla de San Benito como rectora de la observancia de la casa, con ocasión de un precepto de inmunidad obtenido de Luis el Piadoso ${ }^{2}$, por mediación del conde Rampo, con el consiguiente derecho de libre elección del abad y la obligación de gobernar la casa según la Regla de San Benito en lo que es su primera cita de toda la Península Ibérica, según opinión de Lambert ${ }^{3}$. La benedictización es notablemente anterior en los condados catalanes que en el resto de la Península lbérica, donde dicho proceso fue interrumpido por la invasión árabe. Como en tantos otros aspectos, Cataluña mantuvo los lazos de unión con Europa, fruto de lo cual fue la temprana introducción de la regla de San Benito, ampliamente constatada en la primera mitad del siglo IX.

Proceso similar es el que acontece con ocasión de la reforma cluniacense. La escritura fundacional de Cluny en el 910 supone el inicio de un proceso imparable de expansión del nuevo movimiento benedictino. En años tan tempranos como la década de los 40 del siglo $x$ ya se encuentra en la parte sur de los Pirineos con la fundación de San Pedro de las Puellas y de Santa Cecilia de Monserrat, para expandirse por los condados catalanes en la segunda mitad del citado siglo. Veremos posteriormente la importancia que tiene el benedictismo para el correcto entendimiento y ubicación histórica del epigrafe objeto de estudio.

La inscripción en cuestión es una lápida de terracota de forma rectangular, con unas medidas de 56 centímetros de alto, 41 de ancino y 6 de grosor. Los dos laterales del soporte se encuentran realzados, aunque el realce del lado derecho está prácticamente perdido. Junto a ambos realces, hacia la parte interior hay una decoración formada por tres surcos a cada lado, a modo de molduras, realizados probablemente con la punta de un dedo cuando la arcilla aún estaba tierna. Decoración similar, aunque con forma semicircular, se sitúa en la parte superior de la inscripción justo sobre el texto escrito. Son unos motivos decorativos de origen romano, pues se trata de un soporte reaprovechado, en concreto de una tégula de una cronología aproximada del sigio $v$. En el próximo castillo de Porqueras ha sido

\footnotetext{
3 Citado por Constans I SerRats, o.cit, vol. I, p. 6. A. Linage, "La implantación de la regla benedictina en los condados catalanes" en Tiempo de monasterios. Los monasterios de Cataluña alrededor del año mil, Barcelona, 2000, p. 48.
} $147-150$

L.G. Constans I Serrats, Diplomatari de Banyoles, Banyoles, 1985, vol. I, documento 1, pp. 


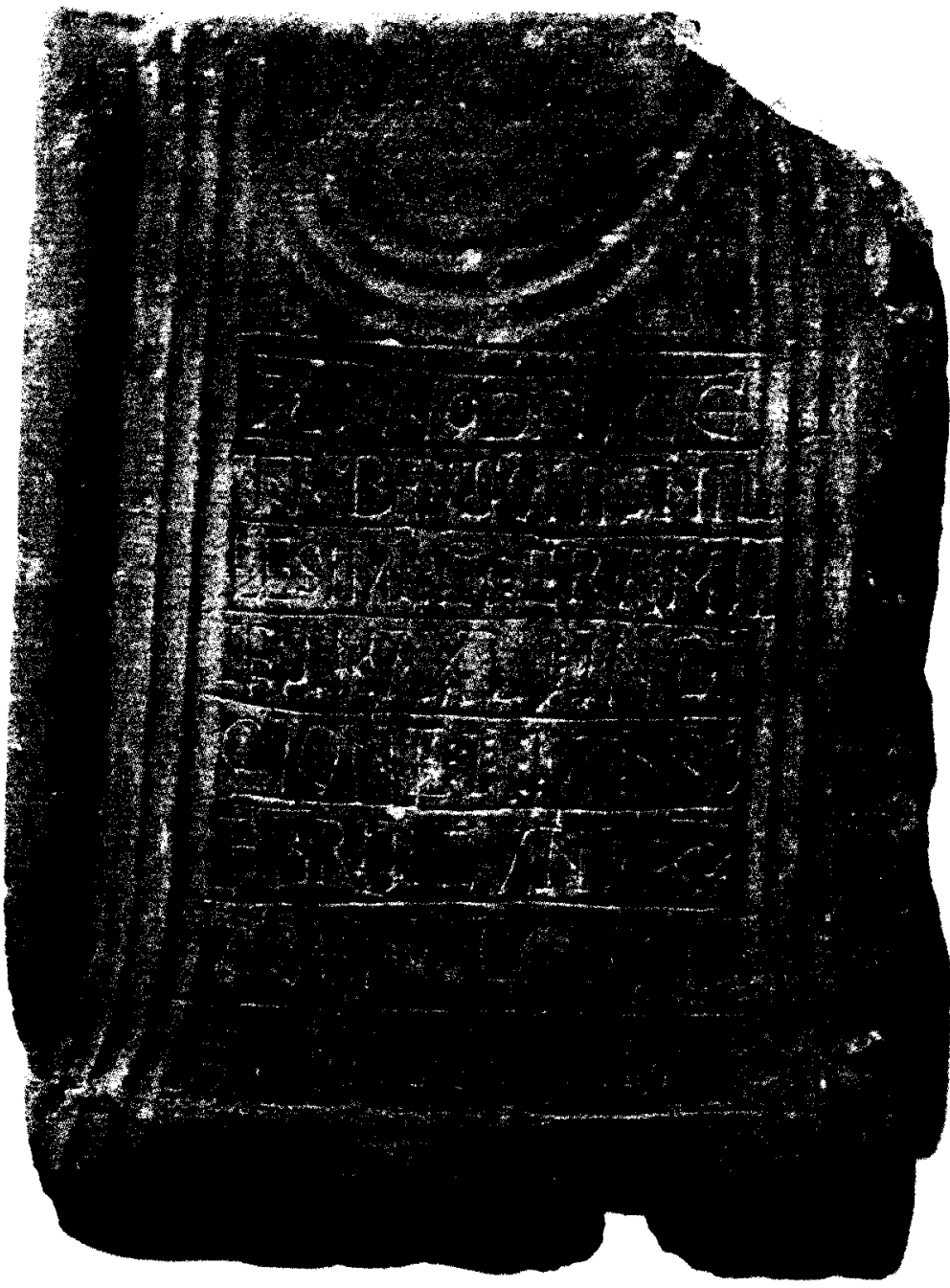

hallada una necrópolis que ha permitido el conocimiento de numerosos sepulcros exactamente iguales al soporte de la inscripción, con idéntico aspecto, material y decoración. También parece haber noticias de un cementerio similar, con parecidos restos, en el paraje de los alrededores del lago de Bañolas llamado Lió ${ }^{4}$. De cualquiera de estos dos sitios pudo pro-

4 Marqués Casanovas, art. cit, pp. 25-26. 
ceder originalmente la tégula que se utilizó como soporte escriptorio. El reempleo de objetos de épocas anteriores como soportes epigráficos es algo relativamente habitual en Cataluña entre los siglos IX y XII. Se conocen otros once epígrafes realizados sobre material reutilizado, entre los que se puede citar el conocidísimo epitafio de Wifredo Borrell, en el monasterio de San Pablo del Campo, en Barcelona ${ }^{5}$. También en las construcciones se empleó con cierta frecuencia material reutilizado.

La escritura está estructurada en ocho renglones que quedan separados unos de otros por unas líneas paralelas a modo de pautado. En la parte superior, individualizando el texto de la decoración, hay dos líneas en lugar de una, según sucede en el resto de la inscripción.

El espejo epigráfico se inicia a algo más de 15 centímetros del borde del soporte para terminar a casi 5 de la parte inferior. La escritura ocupa la totalidad del espacio dispuesto para su trazado, incluso en los renglones segundo, tercero y séptimo lo sobrepasa, montando sobre las molduras del lado derecho, que claramente fueron realizadas con anterioridad a la ejecución de la escritura, según ya indiqué anteriormente. El tamaño de las letras manifiesta cierta irregularidad, con tendencia a aumentar de tamaño en los renglones quinto, sexto y séptimo, para volver a reducirse ligeramente en el último. La altura de esas letras oscila entre 3,5 y 1,5 centímetros aproximadamente. El espacio entre las diversas líneas que señalan el pautado está entre los aproximadamente 3,5-4 cms. del primer renglón y los casi 5 de los dos últimos.

El epígrafe está en buen estado de conservación, aunque fragmentado en la parte superior izquierda y en general en todo el lateral izquierdo, sin afectar dicha fragmentación a la escritura.

$\begin{array}{lll} & \text { ANNO DNICE } & \text { (NN enlazadas) } \\ & \text { TRABEATIONIS MIL } & \text { (TR enlazadas) } \\ 3 & \text { LESIMO * III * ERA MIL } & \\ & \text { LESIMA XLI * INDI } & \\ & \text { CIONE * I* * IIII NNS } & \text { (NN enlazadas) } \\ 6 & \text { FBRII * LVNA XX } & \\ & \text { V* OBITV LVNESI PBRI } & \\ & \text { ET MONACHIVS }\end{array}$

5 E. HüBner, Inscriptiones Hispaniae Christianae, Berlín, 1871., núm. 286. J. VIVES, “Inscripciones prerrománicas barcelonesas", San Jorge, 47 (1962), núm. 47. A.M. MUNDó, Obres completes. I. Catalunya. 1. De la romanitat a la sobirania, Barcelona, 1998., pp. 182-184. J. DE SANTIAGo FERNANDEZ, La Epigrafía latina medieval de los condados catalanes (815-circ. 1150), Madrid, 2003, núm. 54. 
Anno D(omi)nice / Trabeationis mil/ ${ }^{3}$ lesimo III, era mil/lesima XLI, Indi/cione I, IIII $n(0) n(a) s /{ }^{6} f(e) b r(u a r) i i$, luna $X X / V$, obitu(m) Lunesi, $\mathrm{p}$ (res)b(ite)ri / et monachi.

En el año 1003 de la trabeación del Señor, en el 1041 de la Era (1003 d.C.), en el primer año de la indicción, el día 4 de las nonas de febrero ( 2 de febrero), luna 25, murió Lunes, presbitero y monje.

La transcripción de las dos últimas líneas precisa alguna aclaración, por la existencia de ciertas letras y palabras que han sido objeto de rectificación. Al final de la séptima línea, entre la $S$ de LVNES y la P de PBRI se ha introducido una I, añadida con posterioridad al resto de la escritura, de ahí su tamaño más pequeño y la casi inexistencia de espacio entre las tres letras. En el último renglón sobre la $V$ de MONACHVS se realizó una letra, en concreto una I, que intenta rectificar a la primera. Su realización posterior es evidente según se puede apreciar por la mayor profundidad del surco sobreponiéndose al inicial de la $\mathrm{V}$. Asimismo resulta claro que se trata de una letra y no de una incisión accidental del estilo de las que existen en el resto de la superficie de la inscripción; es algo evidente si se observan los dos trazitos que rematan, a modo de ápice, el principal de la letra tanto en la parte superior como en la inferior. Quizá la última letra del séptimo renglón pudiera también haber sido añadida con posterioridad, pero el análisis de los elementos externos del texto no permite afirmarlo con certeza, pues no existe ninguna diferencia ni en el modo de incisión, ni en la profundidad del surco ni en el tamaño de la letra; bien es verdad que ello podría venir motivado por la inexistencia de problemas de espacio, como en el caso de la I de Lunesi, y por no necesitar la rectificación de una letra trazada previamente, como sucede con la $V$ del renglón siguiente.

\section{ELEMENTOS GRÁFICOS}

La inscripción presenta una escritura de ductus natural, como se manifiesta en la diferente ejecución de las letras y en los diversos tamaños que éstas presentan. El estudio externo de la inscripción permite en cierta medida reconstruir el proceso de creación del epígrafe, que en general conocemos gracias a los trabajos de Jean Mallon ${ }^{6}$ y Giancarlo Susini ${ }^{7}$, con aportaciones posteriores de otros investigadores. Las diversas fases expuestas por estos autores pueden ser perfectamente apli-

En especial su Paléographie Romaine, Madrid, 1952

Fundamentalmente en, II lapicida romano. Introduzione all'epigrafia latina, Roma, 1968. 
cadas a la presente inscripción. Ésta manifiesta una evidente preparación previa del soporte antes del trazado de la escritura. No sólo los adornos moldurados que comenté en la parte inicial de este artículo están realizados cuando la arcilla aún estaba fresca, reflejo de la reutilización, sino que las líneas que conforman el pautado también fueron realizadas antes del trazado de la escritura. Esas líneas, al igual que la escritura, fueron ejecutadas claramente a mano alzada, sin ningún instrumento técnico de diseño, como podría haber sido una regula. Así lo deja percibir el hecho de que no sean perfectamente paralelas y el que el ancho de los renglones manifieste cierta oscilación, que en algún caso llega a casi un centímetro. Es la fase previa a la ordinatio según concibió esta última Mallón, una auténtica impaginación con la delimitación del campo de escritura que algunos autores, por comparación con la Codicología y las semejanzas que por ésto adquieren algunas inscripciones con las páginas de un libro, han llamado mise en page ${ }^{8}$.

A continuación se ejecutó la escritura, basándose sobre lo que Mallon llamó minuta, es decir el borrador previo de la inscripción, bien en su redacción ya definitiva, según pensó el investigador francés ${ }^{9}$, o bien simplemente con los datos esenciales dados al artesano por el cliente o comanditario, esenciales para la posterior inscripción, según opinión de Garcia Lobo ${ }^{10}$. En muchas inscripciones lo más probable es que existiese una fase intermedia entre la minuta y la incisión definitiva de la escritura, la llamada ordinatio, consistente en una transliteración de la minuta al soporte definitivo, en este caso el tablero de terracota. Consiste esta fase en un diseño previo, realizado con un carboncillo, tiza o punta seca, que supone el trazado de las letras previo a la incisión ya con el ductus y aspecto definitivo que tendrán. Ha habido autores que han dudado de la existencia de esta etapa en todas las ocasiones, pensando que era algo reservado para las inscripciones más solemnes, como fue el caso del $P$. Ferrua ". Al respecto, creo que la opinión más acertada es la de Susini, quien considera que las generalizaciones son peligrosas, aunque lo más probable es que en la mayor parte de las ocasiones existiese, y que quizá

8 Así MALLON, "Pour une nouvelle critique des chiffres dans les inscriptions latine gravées sur pierre», Emerita, 16 (1948), pp. 14-45; R. MARICHAL, "Paléographie et Epigraphie latines» en Actes du deuxiéme Congrès d'Epigraphie grecque et latine, París, 1953, pp. 181-182; V. García LoBo, "La Epigrafía Medieval. Cuestiones de método" en Centenario de la Cátedra de "Epigrafia y Numismática", Universidad Complutense de Madrid, 1900/01-2000/01. Madrid, 2001, p. 87.

MALLON, "Scriptoria Epigraphiques", Scriptorium, 11 (1957), p. 179, nota 3.

10 Garcia Lobo, "La Epigrafía Medieval. Cuestiones de método", p. 93.

11 Citado por Susini, o. cit, p. 19 y $44-46$. 
haya que entender como ordinatio la acción de transportar a la piedra el texto previo de la minuta, sin estar siempre clara la distinción entre la ordinatio y la incisión ${ }^{12}$.

En la inscripción que estamos analizando la preparación previa es clara, según se dijo anteriormente. Ahora bien, el borrador que antecede a la escritura plantea serias dudas acerca de su existencia. La espontaneidad parece evidente, como se deriva de las diferencias de ejecución de las diversas letras. En el mismo sentido apunta la incapacidad del artesano para adaptarse al espacio disponible, lo cual ha derivado en sobrepasar el marco escriptorio en los renglones segundo, tercero y séptimo, especialmente en este último, donde nada menos que tres letras han quedado fuera del espacio originalmente dispuesto para la escritura. El mismo descuido en la disposición del texto queda patente en la irregular distribución de las letras en los diversos renglones. Así la l.1 tiene 9 letras, la I.2 15, la I.3 15, la I. 413 , la I.5 13, la I.6 11, la I.7 16 y la I.8 sólo 10. Esa deficiente disposición es la que motiva la notable variedad de tamaño entre la altura de las letras mayores y las más pequeñas, pues por ejemplo en la l.6 las dos XX finales tuvieron que ser reducidas para encuadrarlas en su espacio, solución que ya no fue posible en el renglón siguiente en el que tres letras quedaron fuera de él; de igual modo en la 1.8 , donde la $V$ de la palabra LVN ha sido reducida de tamaño como medio de ahorrar espacio.

Las mismas dudas acerca de la preparación previa plantean las correcciones constatadas en las dos últimas líneas, a las que ya me refería con motivo de la transcripción. La razón de las rectificaciones obedece al deseo de convertir en genitivo tres palabras originalmente en nominativo; tal caso es obligado para ser gramaticalmente correcto con el uso del vocablo obitum. Para ello se añade una I al final de Lunes y de Pbtr, operación que no ocasiona problema alguno, salvo los ya referidos de espacio para el caso de la I. La conversión de la palabra Monachus en genitivo es más problemática pues hubiera precisado del borrado de las dos últimas letras. La solución refleja el poco cuidado y la escasa familiaridad del lapicida con el trabajo epigráfico, pues en lugar de eliminar esas dos letras, una vez constatado el error gramatical, se limitó a realizar la I sobre $V$ sin suprimir el texto primitivo ${ }^{13}$.

Pese a todo, no obstante los problemas de espacio y el mal cálculo efectuado antes de realizar la incisión, no se cometen errores gramaticales

12 SUSINI, 0. cit, pp. 19-21 y 45-46.

13 Maroués Casanovas, art. cit, pp. 24. 
al cortar las palabras al final del renglón. Es más, la falta de ajuste, ya mencionada, del renglón séptimo viene dada por el deseo de mantener la unidad de la abreviatura $\mathrm{P}(\mathrm{res}) \mathrm{B}(\mathrm{i}) \mathrm{T}(\mathrm{e}) \mathrm{R}$, añadiendo posteriormente la I.

El siguiente, y último, paso viene dado por la ejecución definitiva de la escritura, por lo que se suele llamar incisión. En este caso, a diferencia de to habitual en este tipo de documentos, se ha realizado con un punzón o punta seca, un stylus, arañando la superficie escriptoria, pasando el instrumento repetidas veces hasta lograr por desgaste el surco que da forma a la letra. Se aprecia como el quadratarius ha ido reduciendo la presión realizada por el stylus conforme avanzaba en la ejecución de la inscripción, o mejor ha realizado la operación de desgaste que da lugar al surco en menor número de ocasiones. El resultado es que las últimas líneas, especialmente la sexta y la séptima, tienen unas letras con un surco notablemente menos profundo. Incluso algunos caracteres del renglón séptimo parecen estar simplemente arañados.

Vistas las diversas etapas es evidente que este epígrafe nos introduce en el interesante tema de los errores en las inscripciones, del que ya se ocuparon con singular acierto Mallon ${ }^{14}$ y Susini ${ }^{15}$. En el caso del epígrafe de Bañolas es complicado conocer a qué fue debido el error. Los equívocos han aparecido en inscripciones muy cuidadas y solemnes y parece que en algunas ocasiones pudieron estar motivados por falta de entendimiento entre las personas especializadas que desempeñaban las diversas tareas en las fases de constitución de una inscripción, que antes hemos revisado. En este caso esa explicación no parece probable. Dado el aspecto general de la inscripción y la inexistencia de taller epigráfico conocido en Bañolas o sus proximidades parece poco probable que en su ejecución participaran diversas personas, lo más probable es que fuera una sola. Debido a ello tampoco se puede aducir ignorancia del lapicida, porque sin duda sería un monje familiarizado o al menos conocedor del arte de escribir. Quizá el error sea reflejo de una simple distracción que le llevó a poner en nominativo lo que debía ir en genitivo o, tal vez, a olvidar escribir la forma correspondiente del verbo sum para consignar el pretérito perfecto pasivo de obeo, con lo que la frase quedaría "obitus est Lunes presbiter et monachus». Es evidente que, dado ese error, resultaba mucho más fácil corregir añadiendo dos letras y sobrescribiendo una tercera que incluir la palabra est en un lugar en el que por espacio era prácticamente imposible. La utilización de abreviatura en el vocablo

14 MALLON, "Scriptoria epigraphiques», pp. 177-194.

15 Susini, O. cit, pp. 57-63. 
OBITV posibilitaría la solución dada. Esto además explicaría el uso del participio pasado para reseñar la muerte, fórmula que no he podido constatar en inscripciones similares, en las que lo habitual es el empleo del verbo en pretérito perfecto.

Parece muy probable que el autor de la inscripción fuese un monje integrante de la comunidad de Bañolas; la autoría clerical está presente en la mayor parte de la producción escrita de los siglos altomedievales. Eran los miembros de la Iglesia los únicos, junto a los jueces, que en la mayor parte de las ocasiones eran clérigos, que utilizaban la escritura en el desarrollo de sus tareas cotidianas.

La técnica y el material empleados han mediatizado la escritura. Es evidente que para conseguir la incisión el quadratarius o lapicida precisa ejercer una fuerte presión, lo cual en algunas ocasiones ha deformado el trazado de la escritura. Es algo que se aprecia en los trazos curvos, más difíciles de conseguir al presionar fuertemente sobre la superficie escriptoria. En algunas ocasiones, en lugar de suaves curvas tenemos trazos rectilineos que se yuxtaponen para formar la letra. Ejemplo magnífico al respecto lo encontramos en las $O$, especialmente en las de la tercera, quinta, séptima y octava líneas.

La escritura utilizada es la monumental típica de este tipo de objetos escritos. No debe olvidarse que la finalidad esencial de cualquier inscripción es la publicidad, conseguir que el mensaje llegue a un público lo más amplio posible, en este caso la comunidad monástica de Bañolas. Esa intención publicitaria suele ir acompañada de un intento de solemnidad, que evidentemente servirá para atraer el interés del posible lector, para llamar su atención e invitarle a la lectura. A esta función también contribuyen los elementos decorativos, aunque fueran reutilizados de algún monumento anterior, y por supuesto el pautado de los renglones. Esto constituye una jerarquización de la escritura, observada desde antiguo, en la que se emplea un tipo determinado dependiendo de la finalidad. Así no sólo los epígrafes utilizaron de modo sistemático la escritura mayúscula, de más fácil legibilidad y mayor solemnidad, también la encontramos en los incipit, explicit, títulos principales y secundarios, prefacios, primeras líneas de texto, iniciales, etc. de otros objetos escritos.

El tipo de escritura empleada es la habitual en esta época y zona, la llamada carolina, asentada y utilizada en los territorios condales de la Marca Hispánica desde mediados del siglo Ix. Esto es resultado del dominio político franco, que supone la introducción de elementos escriturarios que tardarán al menos siglo y medio más en llegar a otros territorios peninsulares. El ambiente de renovación cultural de la Corte franca, 
la dependencia política, con todo el movimiento documental entre la Corte carolingia y los condados que eso supuso, y la incorporación de los territorios a la diócesis de Narbona, con la consiguiente llegada de códices litúrgicos y culturales, así como numerosos monjes y obispos francos con el fin de sustituir el rito mozárabe por el francés o galicano, determinó la introducción de la escritura carolina en las tierras catalanas al norte del Llobregat. La unidad de la escritura era una herramienta necesaria para el desenvolvimiento del nuevo imperio carolingio. Es un proceso de identidad con el norte de Europa que se observa no sólo en la escritura, sino también en otros hechos, como la liturgia o la moneda, pues no debe ser olvidado que Carlomagno llegó a emitir moneda en los territorios condales.

La escritura de la inscripción aquí estudiada es un magnífico ejemplo del uso de la carolina. En todo momento muestra su raíz clásica, con muchas formas que encajan perfectamente con el aspecto de la vieja escritura latina, propia de las inscripciones de la antigua Roma, aunque como es lógico, también con formas nacidas a partir del siglo IV. Se trata de una escritura austera, ajena a los principios ornamentales que aparecen en otras zonas de la Península, especialmente en el grupo epigráfico mozárabe. La mayor parte de las letras presenta a modo de remate de los trazos, una pequeña rayita o ápice, generalmente en perpendicular al trazo principal, que está intentando recordar los típicos remates triangulares, tan frecuentes en las inscripciones de época romana y que habían pervivido en tantas inscripciones medievales. La naturalidad en la ejecución del ductus, la dureza del soporte y la técnica de ejecución por desgaste y no por incisión con martillo y punzón determinan que esos triángulos se hayan convertido en simple rayitas.

Las diversas formas gráficas constadas presentan la siguiente ejecución o ductus (ver fig. 1):

A: La A presenta tres modos de ejecución. La forma más repetida, en cuatro ocasiones, se caracteriza por un modo de hacer la letra a partir de cinco trazos, con el travesaño en $\mathrm{V}$, manteniendo un modo de realización enormemente popular en la epigrafía de época visigoda y en general en gran número de inscripciones europeas de los primeros siglos de la epigrafía cristiana. En el ángulo superior de unión de los dos trazos laterales se realiza otro en horizontal, aunque éste no se sitúa en el centro del ángulo, sino que está desplazado hacia la izquierda, lo cual es resultado de la naturalidad del ductus y de la espontaneidad en la ejecución. También es una forma conocida tanto en Cataluña como en la el resto de la Peninsula. 


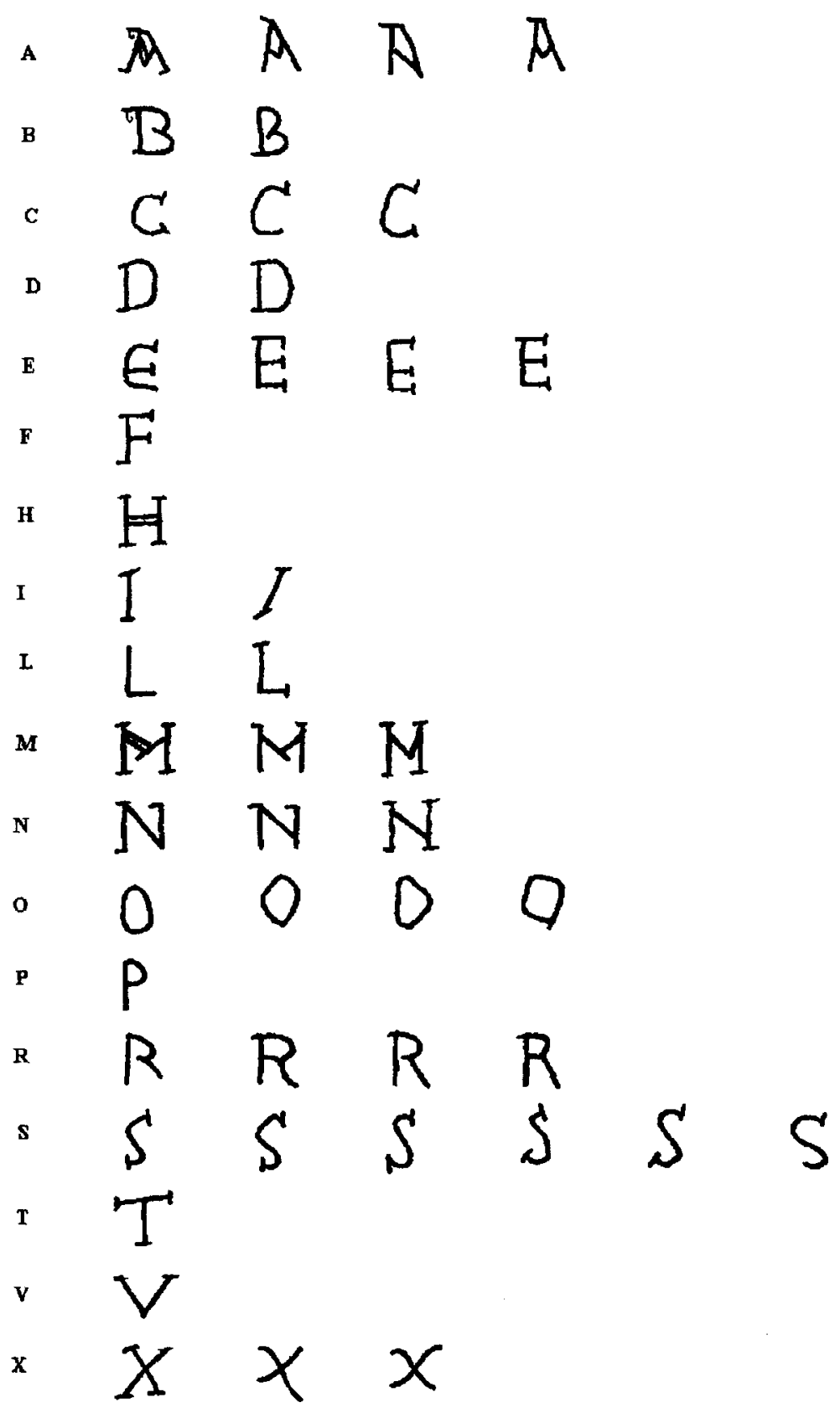

Figura 1. 
Directamente relacionada con la anterior forma está una $A$ en cuya ejecución el primer trazo del travesaño en $V$ se ha hecho doble, característica peculiar en el panorama epigráfico catalán de esta época, especialmente en el siglo XI, y que es algo típico de la escritura carolina. Encontramos este tipo de ductus en dos ocasiones. En una de estas dos A quiero mencionar una característica curiosa que es una especie de bucle que puede recordar la forma de ejecución de la T visigótica en otras zonas de la Península, no en Cataluña, donde no ha sido constatada más que en ejemplares aislados, como el epitafio de Gescafredo ${ }^{16}$, procedente de Barcelona, aunque quizá realizada por algún artesano originario de tierras gerundenses ${ }^{17}$.

El último tipo de A constatado, empleado sólo en una ocasión, difiere en la realización del travesaño, ejecutado con un sólo trazo de fuerte inclinación hacia la derecha. Esa inclinación no parece ser sustancial a la letra, sino más bien resultado de la espontaneidad a la hora de realizar la escritura.

B: La B presenta dos formas de ejecución. La mas frecuente, presente en tres ocasiones, cuenta con tres trazos. En ella, el trazo que forma el bucle superior acaba en línea recta sobrepasando el punto de unión con el bucle inferior. La parte superior e inferior de ambos bucles es también recta. La terminación de los trazos que forman los bucles no se junta con el trazo vertical.

El otro tipo presenta dos bucles más anchos. El ángulo de unión de sus trazos, como en el caso anterior, tampoco se une al trazo vertical. Esta letra presenta un ápice similar al constatado en el caso de la A que, en cierto modo, recuerda la $T$ de bucle propia de la visigótica.

C: Se presenta en tres variantes distintas de un mismo modo de ejecución. Se caracteriza por tener un sólo trazo redondeado que cierra más o menos los extremos.

D: Se ejecuta siempre de acuerdo a la forma capital clásica, en dos trazos. La única diferencia es que en una ocasión el segundo trazo en su parte superior está más redondeado y en otra adquiere cierta rectitud. En ambos casos, la parte inferior de la panza es más o menos recta.

E: Presenta dos modos de ejecución. La más frecuente, constatada en siete ocasiones, es la $E$ de tipo clásico con cuatro trazos rectos, con la única

\footnotetext{
16 HUBNER, Inscriptiones Hispaniae Christianae. Supplementum, Berlin, 1900, núm. 522. J. VIVES, “Inscripciones prerrománicas barcelonesas», San Jorge, 47 (1962), núm. 27. Catalunya Romànica, vol. XX, Barcelona, 1992, p. 247. SANTIAGO FERNÁNDEZ, o. cit., p. 227.

17 Santiago Fernandez, o. cit.
} 
diferencia de prolongar más o menos alguno de los ellos y realizar la letra con distinta separación entre los mismos. En todos los casos, salvo uno, tanto el trazo superior como el inferior superan por la izquierda el vertical.

El segundo modelo viene dado por la típica $E$ uncial de dos trazos, el primero de ellos redondeado, aunque en este caso la técnica inscriptoria y la dureza del material, además de la espontaneidad del trazado, han determinado que su parte inferior adquiera una forma más rectilínea, que indica su ejecución en dos tiempos.

F: Está ejecutada a la manera clásica, realizada con tres tramos rectos. El central es algo más corto y el superior supera por la izquierda el vertical.

H: Es la $\mathrm{H}$ de tipo capital clásico, con la única particularidad de que el travesaño central está formado por un doble trazo, en una caracteristica similar a la testimoniada para uno de los tipos de $\mathrm{A}$, que la vinculan de modo evidente a la escritura carolina.

I: Es la I clásica, resuelta con un único trazo vertical, aunque tiene variantes en la disposición y modo de ejecución de los ápices, variaciones que no parecen deberse a otra cosa más que a la espontaneidad a la hora de realizarla.

Un segundo modelo tiene inclinación a la derecha, destacando la oblicuidad del ápice inferior. De cualquier modo, es una letra un tanto especial, puesto que se escribe sobre otra letra, una $V$, a modo de corrección y quizá pretende mostrar claramente esa superposición y corrección, lo que lleva al lapicida a inclinarla para evitar una confusión con una letra enlazada o encajada.

L: Presenta dos tipos, realizados ambos con dos trazos. El más abundante cuenta con ápices al final de los trazos y el segundo sobrepasa por la izquierda al vertical. El otro tipo carece de ápices.

M: La M tiene tres tipos, los tres emanados y variantes de la de época clásica y, por tanto, con cuatro trazos, todos ellos rectos y con los dos exteriores paralelos. La primera de ellas, repetida en tres ocasiones, tiene su característica más destacada en el segundo tramo, realizado con doble trazo, de una manera similar a como ya se señaló para la $A$ y la $H$, que como dije vincula claramente la inscripción a la escritura carolina. Una M en cierto modo parecida se utiliza en el epitafio de Arnulfo ${ }^{18}$, procedente

18 A. Pladevall, "Arnulfo, abat de Ripoll i bisbe de Girona», Revista de Girona, 83 (1978), pp. 149-155. Catalunya Romànica, vol. V, Barcelona, 1991, p. 32. SANTIAGo Fernández, o. cit., núm. 36. 
de la catedral de Gerona, y correspondiente de forma probable al siglo xi o quizá algo posterior dada la escritura utilizada. El segundo trazo de los dos centrales se inicia, no en el comienzo del vertical, sino más hacia su parte central; asimismo remata superado el vértice de unión con el segundo trazo, pero sin llegar hasta la línea del renglón.

El segundo tipo es similar pero sin el doble trazo reseñado en el anterior. Asimismo en este caso es el primero de los dos trazos centrales el que sobrepasa el vértice de unión, como antes sin llegar a la línea del renglón. Los dos trazos centrales no se inician en el extremo de los laterales, sino hacia el cuerpo de éstos.

Por último, un tercer tipo cuya única diferencia con el anterior viene dada por la unión de los dos trazos centrales, ahora en ángulo perfecto, sin que ninguno de los dos tramos lo sobrepase. De cualquier modo, esto no son concepciones diferentes de una misma letra, sino distintos terminados derivados de la espontaneidad de trazado.

$\mathrm{N}$ : Cuenta con tres tipos, todos ellos realizados con tres trazos rectos. El más frecuente, repetido en ocho ocasiones, se caracteriza por iniciar y rematar el trazo inclinado en el cuerpo de los laterales.

El segundo, presente sólo una vez, tiene su caracteristica principal en iniciar el trazo inclinado en el comienzo del lateral, para acabar no sobre la línea del renglón sino en el cuerpo del segundo trazo lateral. El tercer tipo, constatado en dos ocasiones, es la $\mathrm{N}$ clásica, rematando el trazo central en los extremos de los laterales.

O: La ejecución de la O está mediatizada por la dureza del material. En todas las ocasiones el intento es realizarla en un único trazo que se inicia en la parte inferior del renglón, lo que da a la letra un aspecto apuntado hacia su parte baja. Sin embargo, algunas veces la dureza del material impide rematar la letra de un sólo trazo y se ha acabado mediante un último más o menos recto. Además de ello varían en la anchura y en la mayor o menor redondez.

P: No presenta mayor peculiaridad. Se trata de la $P$ clásica resuelta mediante dos trazos.

R: Las cuatro $R$ presentes denotan cuatro tipos, lo cual dice mucho acerca de la espontaneidad de la escritura. Tres de ellas están realizadas con tres trazos y la última con cuatro. La primera de ellas tiene el tercer trazo ligeramente curvo descendente cóncavo hacia la derecha y su punto de unión con el segundo trazo no se ubica en el cuerpo del primero. 
La segunda tiene el tercer trazo ondulado. Éste se inicia en el cuerpo del vertical, mientras que el segundo, el que forma la panza de la letra, remata en el cuerpo del tercero y sobrepasa por la izquierda al vertical.

El tercer tipo se caracteriza por un último trazo recto que forma ángulo con el remate de la panza, pero sin unirse al tramo vertical. El trazo de la panza, a su vez, sobrepasa por la izquierda al vertical.

El cuarto modelo tiene un trazo recto en la parte superior de la panza, formada por éste y por un trazo curvo que se une en ángulo con el tercero, también recto, prolongando dicha unión hasta el cuerpo del tramo vertical.

S: Presenta su forma básica en tres trazos. Las diferencias vienen marcadas por la espontaneidad en el trazado que determina disparidad en la longitud de cada uno de ellos, una mayor o menor estilización y unos arcos superior e inferior más o menos grandes, dependiendo de los casos. En una ocasión la dureza del material ha supuesto un trazo recto en la parte superior, dando lugar a un ángulo más que a un arco. En otra, el arco inferior se halla compuesto de dos trazos rectos yuxtapuestos, en lugar de uno curvo.

T: En todos los casos es la T clásica de dos trazos rectos.

V: De nuevo es una letra clásica, formada por dos trazos. Se emplea exactamente con la misma forma tanto para el valor vocálico como para el consonántico.

$\mathbf{X}$ : Presenta dos formas. La primera es más clásica, en forma de aspa con sus dos trazos rectos. La otra tiene una mayor influencia de la cursiva, pues los dos trazos son hondulados.

Como vemos, en conjunto la característica principal de la escritura es su clasicismo. Apenas aparecen formas unciales, tan sólo uno de los tipos de la $E$, y no se introduce ninguna forma de minúscula agrandada, según suele ser habitual en otras inscripciones, incluso de esta misma zona y época. Es por tanto un tipo de escritura que se adapta perfectamente a los ideales de Carlomagno de retorno a la antigüedad.

Las letras enlazadas o nexos son escasos. En dos casos se emplea el nexo NN y en otro TR. El primero parece un recurso del ordinator para todas las palabras que presentan la doble $\mathrm{N}$, como es el caso de anno y la abreviatura de $\mathrm{N}(0) \mathrm{N}(\mathrm{a}) \mathrm{s}$. El uso de los enlaces es algo habitual en la producción epigráfica medieval y ha sido ampliamente constatado en el estudio de otros conjuntos epigráficos en aquellos casos que ha existido preo- 
cupación gráfica, como podrían ser las inscripciones oscenses ${ }^{19}$, las de San Miguel de Escalada ${ }^{20}$ o las de Zamora ${ }^{21}$, entre otros.

La escasez de nexos enlaza perfectamente con el aspecto general de la inscripción, pues suele ser una característica más vinculada con epígrafes cuidados, realizados con una ordinatio previa, que cuida el aspecto externo de la inscripción y utiliza dicho recurso gráfico para lograr una más perfecta adaptación al marco escriptorio. En esta inscripción vemos perfectamente que es un recurso que no domina bien el autor, por cuanto en algunos renglones sobrepasa el espacio destinado a la escritura, cuando ese problema hubiese podido ser resuelto por medio del enlace de algunas letras. En la misma línea está la ausencia de letras encajadas que sí se constatan en otros epígrafes de esta zona y cronología.

Las abreviaturas empleadas son las siguientes:

DNICE: Dominice

NNS: Nonas

FBRIl: Februarii

PBRI: Presbiteri

Todas ellas van indicadas por el correspondiente signo abreviativo, consistente siempre en una raya sobrepuesta que sigue siendo habitual en el período de la carolina.

Cuenta la inscripción con interpunciones consistentes en tres o cuatro puntos alineados en vertical. Su uso parece querer separar los numerales y las diferentes fórmulas cronológicas. Así las encontramos en el renglón 3 separando el numeral III y la datación por el año de Cristo de la de la Era. En la línea 4 para independizar la indicción y la Era y en el 5 individualizando el numeral I de la indicción y el IIII de las nonas. En este último renglón es curioso, por inusual, el empleo de una doble interpunción entre el numeral de la indicción y el del día de nonas. En la línea 6 también se utiliza interpunción a modo de separación de las dos formas de indicar el día del mes, el de la calendación y el de la edad de la Luna. Por último, en la siete se emplea para separar el día de la luna de la fórmula notificativa.

19 A. DuRÁn Gudıol, "Las inscripciones medievales de la provincia de Huesca", Estudios de Edad Media de la Corona de Aragón, VII (1967), pp. 45-153.

20 Garcia Lobo, Las inscripciones de San Miguel de Escalada. Estudio crítico, Barcelona, 1982.

21 M. Gutiérrez Álvarez, Zamora. Estudios, vol. 1/2 del Corpus Inscriptionum Hispaniae Mediaevalium, Brepols-León, 1999. 
Hubiera sido útil tener la posibilidad de relacionar el presumible taller epigráfico de Bañolas con otros conocidos en Cataluña, por acreditar unas relaciones culturales, comerciales o de cualquier otro tipo. Sin embargo, los datos a través de un único documento epigráfico son demasiado exiguos para poder ofrecer conclusiones con una base lo suficientemente firme. Dentro de la comarca de Bañolas, circunscrita, según criterios geográficos, económicos y monacales, al territorio delimitado por el norte por el pueblo de Sallent hasta Parets del Ampurdán siguiendo el curso de los ríos Ser y Fluviá, por el este desde Parets hasta Vilafreser a través del curso de la antigua Vía Augusta, por el sur desde Vilafreser hasta la sierra de Rocacorba a lo largo del curso de Revardit y Rocacorba, y por el oeste desde Rocacorba a Sallent, a lo largo de las sierras de Rocacorba y Finestres, no existe otro epígrafe salvo el aquí estudiado. Podría pensarse en alguna relación con San Pedro de Roda, priorato dependiente de Bañolas. Allí sí se ha testimoniado algún resto epigráfico, especialmente el epitafio de Tassi, que ha sido relacionado con las inscripciones procedentes de San Martín de Ampurias y Santa María de Rosas por cuestiones escriturarias y de estilo de versificación ${ }^{22}$. Esas tres inscripciones se encuentran cronológicamente situadas entre el 925 y el 955 , lo cual las aleja demasiado en el tiempo de la de Bañolas como para poder efectuar afirmaciones seguras. También varía el material, mármol en las tres inscripciones citadas y terracota en la de Bañolas. Asimismo presenta diferencias la escritura, como también difieren los enlaces utilizados. Como vemos no existe nada en el análisis epigráfico que permita vincular la inscripción de Bañolas con el taller que realizó las otras tres. Tampoco las inscripciones procedentes de Gerona, relativamente próxima a Bañolas, donde se ha podido identificar otro taller epigráfico ${ }^{23}$, parecen tener nada en común con ésta. La conclusión necesaria a esta ausencia de semejanzas y a estas incertezas es que probablemente esta inscripción fue realizada en el mismo monasterio de San Esteban de Bañolas, manifestando autonomía e independencia de los otros dos talleres indicados.

\section{ELEMENTOS INTERNOS}

Esta inscripción es un epitafio. En concreto se trata de una inscripción necrológica que hay que diferenciar de las propiamente sepulcrales ${ }^{24}$. La

\footnotetext{
22 Mundó, "Les inscripcions de Tassi i d'Hildesind de Sant Pere de Rodes segons Marca i Pujades" en Homenaje a Jaime Vicens Vives, Barcelona, 1965, pp. 297-298.

23 Santiago Fernández, o. cit.

24 García Lobo y E. Martín López, De Epigrafía Medieval, León, 1995, p. 39.
} 
diferencia estriba en que las necrológicas, como sucede en ésta de Bañolas, únicamente dan noticia de la muerte de una persona, pero no indican que allí se encuentren depositados sus restos, que es lo que ocurre en las sepulcrales. Este tipo de epígrafes guardan una profunda relación con la costumbre existente en los monasterios de orar por sus deudos y sus benefactores. En esa línea han sido relacionadas con los obituarios existentes en tantos monasterios ${ }^{25}$. Su función principal era recordar a la comunidad monástica la fecha de la muerte de algún personaje significativo para el monasterio con el fin de orar por su alma con ocasión de su aniversario. Probablemente la influencia de la abadía de Cluny tenga que ver con la difusión de este tipo de inscripciones, dado que en esta abadía se instituyó en el año 998 la fecha de la Conmemoración de todos los fieles difuntos ${ }^{26}$, dando lugar a una importante liturgia funeraria.

En algunos monasterios y centros religiosos se ha dado tal concentración de esta clase de epígrafes que algún autor ha hablado de «segundas copias en piedra de un necrologium u obituarium de un centro eclesiástic0» ${ }^{27}$. Sería el caso, por citar algún ejemplo suficientemente conocido, de los conjuntos de la catedral de Roda ${ }^{28}$, de Santo Domingo de Silos, datado en los años centrales del siglo $x \|^{29} \mathrm{O}$ el existente en el monasterio de San Juan de la Peña, cuya cronología se sitúa entre los siglos XIII y $\mathrm{xV}^{30}$. Como vemos son conjuntos epigráficos más tardíos que nuestra inscripción, de datación bastante temprana para lo que suele ser habitual. De hecho, la mayor abundancia de inscripciones de este tipo corresponde al siglo XIII, para ir desapareciendo en el siguiente. El presente epígrafe parece ser el más antiguo de los conservados en Cataluña, lo cual no es extraño debido a su antigua fundación y a que estamos ante un monasterio benedictino, los cuales, como antes se dijo, cultivaban fuertemente la liturgia funeraria. Este anticipo en los condados catalanes de lo que luego sucederá en el resto de la Península se debe, al igual que tantas otras cosas, a la influencia europea, a la intercomunicación con el otro lado de los Pirineos que se deriva del dominio político que tuvieron los francos carolingios.

25 García lobo y Martín López, o. cit, p. 39.

26 R. FavreaU, "L'épigraphie comme source pour la liturgie" en FavREAU, Études d'épigraphie médiévale, Pulim, 1995, pp. 433-434.

27 DURÁN GudIOL, 0. cit, p. 47.

28 DuRAN Gudiol, o. cit, pp. 65-109, núms. 80-269.

29 Garcia Lobo, “La epigrafía del claustro de Silos» en El Románico en Silos. IX Centenario de la consagración de la iglesia y claustro, 1088-1988, Abadía de Silos, 1990, pp. 85-104.

30 DuRan Gudiol, o. cit, pp. 34-55, núms. 5-68. 
Este tipo de inscripciones normalmente estaban en el claustro o en algún lugar del templo fácilmente visible. Ésta de Bañolas fue hallada próxima al presbiterio, en las cercanias del ábside románico y dos absidiolos laterales y en las cercanías de diversas sepulturas. Pese a ese contexto, dudo mucho que la presente inscripción, dado su carácter necrológico, fuese utilizada para cubrir o indicar el sepulcro; ese tipo de inscripciones tienen fórmulas diferentes que indican claramente que alli están depositados los restos del difunto. Parece más probable que hubiese ocupado algún lugar preeminente en las proximidades de la cabecera de la iglesia, donde pudiese ser fácilmente contemplada por la comunidad.

Con estos antecedentes debemos concluir que el personaje honrado hubo de tener importancia y relevancia para el monasterio. En este caso, sabemos que hubo un Luneo del que tenemos referencias como abad en el 928, mencionado en un documento de permuta de bienes datado el 23 de julio de ese año ${ }^{31}$. Dado que la inscripción dice de forma inequívoca que murió en el año 1003, parece evidente que no se trata de la misma persona. El nombre es infrecuente, pero no desconocido, en la Cataluña de la época. Se llamó Lunesio el primer abad de Santa María de Rosas, correspondiendo a una datación de en torno al 960, se constata, bajo la forma Lunes y Luneus, en una carta de donación del año 1057 y se observa que en la forma Lunes y Llunes era usado en el siglo XIV. Sin duda fue un monje perteneciente al monasterio, según parece acreditar un documento datado en el 1009, la respuesta del monasterio de Bañolas a la encíclica anunciadora de la muerte de Sunifredo, abad de Ripoll, el 4 de julio del $1008^{32}$. En él se hace un ruego de oración por diversos personajes, entre los que se cita a Lunes. Parece muy probable que se trate del protagonista de nuestra inscripción. Más difícil resulta conocer cuáles fueron los méritos que le hicieron acreedor del recuerdo de su muerte en una forma tan solemne y notoria como es un epígrafe. En el documento antes citado se habla de los protomartyris Stephani Valneolas, lo cual puede ser alusivo a los personajes después mencionados entre los que se encuentra Lunes. Probablemente fue un monje de vida ejemplar, cuyo recuerdo pudo servir de modelo de conducta a los monjes venideros. De hecho, la inscripción necrológica sirve para conmemorar el aniversario de la muerte.

La estructura interna de esta inscripción se adapta perfectamente a lo que suele ser común en los epígrafes necrológicos. En primer lugar la data, en éste indicando el año y el día concreto del óbito, que es el elemento cro-

\footnotetext{
Constans I Serrats, o.cit, vol. I, documento 29.

Constans I Serrats, o.cit, vol. I, documento 53.
} 
nológico que nunca falta, dada la función conmemorativa y de recuerdo, además de litúrgica, que tienen estas inscripciones. A continuación figura el verbo notificativo, normalmente obiit, aunque en esta ocasión ha sido sustituido por el participio pasado. Por último, la intitulación, con el nombre y los cargos del difunto, quien acredita haber sido presbiter y monachus.

La cuestión de la data es interesante. El año ha sido constatado utilizando tres cómputos diferentes, la era de la Trabeación, la era hispánica y la indicción. La triple data es relativamente habitual en la documentación catalana de la época de tipo solemne, y la producción epigráfica indudablemente lo es, aunque el año de Cristo no suele ser indicado mediante la Trabeación. Es alto el número de inscripciones catalanas de los siglos ix al XII que emplean el triple cómputo, nada menos que diez, incluyendo ésta de Bañolas, lo cual constituye el $36 \%$ del total de inscripciones datadas existentes. Esta característica de los epígrafes catalanes, y por añadidura del de Bañolas, sólo puede ser entendida en el contexto de las especiales influencias, políticas, culturales y religiosas, a las que se encuentran sometidos los territorios de la Marca Hispánica, mucho más relacionados con el norte de Europa que el resto de zonas peninsulares, en las que lo habitual es la simple datación por la era hispánica. Es por tanto habitual la multiplicidad de cómputos; sin embargo no lo es tanto el orden en el que aquí han sido constatados. Normalmente en las inscripciones catalanas la era hispánica siempre precede a la cristiana. Ésta es la única inscripción procedente de los antiguos condados catalanes en la que esa precedencia ha sido alterada y se ha situado el año cristiano delante del ofrecido por la era.

La era de la Trabeación es uno de los estilos empleados en la era cristiana. La forma de datar de acuerdo a los años de Cristo se introdujo en los estudios de escritores y cronistas antes que en las oficinas de documentos. En Francia ya se conocen algunos en el siglo VIII, pero es documentación de tipo privado. En las inscripciones no se hace habitual antes del año 1000 , aunque desde el siglo Ix se constatan diecinueve ejemplos, siendo el más antiguo el epitafio de Adelberge, procedente de San Martín de Tours, datado en el $830^{33}$. En la Península Ibérica el ejemplo epigráfico más antiguo, que yo conozca, es el epitafio de Witiza ${ }^{34}$, datado en el 900 , que inicia

33 Favreau, Epigraphie médiévale, Turnhout, 1997, p. 174.

34 HüBNER, Inscriptiones Hispaniae Christianae, núm. 286. J. AINAud, J., Gudiol, y F.P. VerRIE, Catálogo monumental de España. La ciudad de Barcelona, Madrid, 1947, p. 153. VIVES, art. cit., núm. 47. Mundó, Obres completes, pp. 188-189. SANTIAGo FERnÁNDEZ, “Estudios documentales de Epigrafía y Numismática. En la epigrafía catalana altomedieval y el velión castellano de la Edad Moderna" en l Congreso universitario de Ciencias de la Documentación en España (1975-2000), Madrid, 2000, pp. 743-749. SANTIAGo FERNÁNDEZ, La epigrafía latina medieval de los condados catalanes, núm. 53. 
la serie de epitafios barceloneses que portan la data cristiana. Otros conjuntos epigráficos peninsulares conocidos ofrecen las siguientes fechas. En la catedral de Roda el primer testimonio corresponde a mediados del siglo XIII; se trata del epitafio de tres obispos, cuya fecha explícita es el 1225; sin embargo el análisis de la escritura demuestra que esta inscripción es contemporánea de las del necrologium del claustro, grabado alrededor del $1240^{35}$. En la catedral de Jaca el epitafio de Eximino de Exea, del 1276, ya está datado por la era cristiana ${ }^{36}$. En San Juan de la Peña, la primera inscripción datada por el anno Domini es el epitafio de Bartolomé del $1370^{37}$. En la provincia de Zamora, la consagración de la catedral, del año 1174, se fecha por el año de Cristo ${ }^{38}$, pero estamos ante un caso aislado, pues no volvemos a encontrar una inscripción original con dicho cómputo hasta mediados del siglo xIv, en la datatio del monasterio de Moreruela, en la que se lee explícitamente anno ab Incarnacione ${ }^{39}$, aunque eso no suponga la imposición definitiva de este modo de datar, pues se conservan epígrafes posteriores que aún utilizan la era, como es el epitafio de don Rodrigo Yáñez, correspondiente a finales del siglo XIV o principios del $X V^{40}$. En Asturias el primer epígrafe con la era cristiana es el epitafio del abad Rodrigo, procedente del monasterio de San Vicente, en Oviedo, del $1178^{41}$, pero de nuevo estamos ante un caso aislado frente al casi absoluto predominio de la era hispánica; el cofre de San Eulogio, del 1305, lleva explícita la fórmula anno Domini ${ }^{42}$. A partir de ese momento, en Asturias, las fórmulas cronológicas empiezan a introducir la datación por el año de Cristo, aunque aún con predominio de la era. En el monasterio de San Miguel de Escalada tenemos dos inscripciones ${ }^{43}$ relativamente tempranas, fechada una en 1261 y la otra en 1287, que se explican en función de la influencia del elemento francés en la comunidad de Escalada, dada la donación que

35 DURÁN GUDIOL, art. cit, núm. 79.

36 DURÁN GUDIOL, art. cit, núm. 76.

37 DURAN GUDIOL, art. cit, núm. 64. No he tenido en cuenta la inscripción de Lope Ferrén (núm. 54), presumiblemente del 1301, por no tener la seguridad de que la data corresponda al año de Cristo, al no mencionarlo la inscripción y estar todas las siguientes hasta el citado 1370 datadas por la era hispánica. De cualquier modo, caso de obedecer su datación a la era cristiana, sería un caso absolutamente aislado en fecha tan temprana.

38 Gutiérrez Álvarez, Zamora. Colección epigráfica, vol. l/1 del Corpus Inscriptionum Hispaniae Mediaevalium, Brepols-León, 1997, núm. 25. He obviado el epitafio del obispo Bernardo (núm. 18), por ser una copia moderna de una inscripción necrológica, lo cual hace muy complicado saber si el año consignado corresponde al de ejecución de la inscripción.

39 Gutiérrez Álvarez, Zamora. Colección epigráfica, núm. 88.

40 Gutiérrez Álvarez, Zamora. Colección epigráfica, núm. 99.

41 F. Diego Santos, Inscripciones medievales de Asturias, Oviedo, 1994, núm. 98

42 Diego Santos, 0 . cit, núm. 28.

43 Garcia Lobo, Las inscripciones de San Miguel de Escalada, núms. 27 y 29 
Alfonso VII hizo del monasterio a la abadía de San Rufo de Valance ${ }^{44}$, siendo, además, dos ejemplos aislados, pues todas las demás inscripciones medievales de allí procedentes se fechan por la era hispánica.

En principio, la era Trabeationis constituye el llamado estilo de la Pasión, por el hecho de que supuestamente empieza su cómputo a partir de la fecha de la Pasión, cuestión compleja y difícil puesto que diversos cómputos sitúan tal hecho en el 28,30 y 33 d.C. Además, parece que los scriptores medievales no tenían muy clara la diferencia, pues en la mayor parte de las ocasiones hay confusión entre el anno Domini, en cualquiera de sus estilos, sea el de la Natividad o el de la Encarnación, y el anno Trabeationis, iniciando el cómputo en el nacimiento de Cristo. Al respecto es concluyente, al igual que otros documentos, esta inscripción de Bañolas, pues al estar datada de tres modos distintos permite hacer las preceptivas comprobaciones. Vemos como el año dado por la era hispánica, 1041, una vez restados los correspondientes 38 años, coincide exactamente con el ofrecido por la era trabeationis, 1003. La conclusión evidente es que este cómputo no comienza, al menos en este caso, con el año de la Pasión, sino con el del nacimiento de Cristo.

La indicción, ese período fiscal de quince años empleado a partir del siglo IV en la data de documentos, corrobora lo antes dicho. El año I de la indicción 46 es justamente el 1003. Es una fórmula cronológica escasamente presente en los usos hispanos, tanto documentales como epigráficos, pese a que tres inscripciones de época visigoda sí lo emplean ${ }^{45}$. Diferente es la situación en Francia, donde empieza a utilizarse en los epígrafes a partir del siglo $\mathrm{v}$ y en el primer tercio del $\mathrm{VI}$ ya es un sistema habitual; se mantiene con cierta frecuencia hasta el siglo XII ${ }^{46}$. Estamos de nuevo, por tanto, ante un hecho casi específico de Cataluña, dentro del contexto peninsular, y que es resultado de la fuerte influencia franca en estas tierras.

La indicación del día del mes tiene interés. Ésta se caracteriza por no emplear sólo el tradicional y masivamente usado sistema romano, consistente en la división del mes en tres fechas dadas, como son los días de kalendas, nonas e idus. Es un sistema sobradamente conocido y que no merece mayor comentario. De acuerdo a él, la muerte de Lunes se produjo el 2 de febrero. Mucho más extraño y poco usado en la Epigrafia me-

44 Garcia LoBo, Las inscripciones de San Miguel de Escalada, p. 37

45 Ver M.T. MuÑoz GaRcia DE ITURROSPE, Tradición formular y literaria en los epitafios latinos de la Hispania Cristiana, Vitoria-Gasteiz, 1995, pp. 227-228.

46 Favreau, Épigraphie médiévale, p. 178. 
dieval hispana es la alusión al día en relación con el curso de la Luna, Luna $X X V$ se indica en la inscripción. Es una mención a la edad de la Luna, conocida en documentos entre los siglos $x$ y XII. Consta en algunos documentos catalanes de esa época, como, por citar algún ejemplo, el acta de dedicación de la iglesia del monasterio de Sant Benet del Bagés ${ }^{47}$, la narración de la toma de Barcelona por Almanzor ${ }^{48}$ o la carta de población de Cardona ${ }^{49}$. El numeral XXV está indicando que han pasado veinticinco días desde la última luna nueva, que es el día de inicio del mes lunar, el cual tiene una duración de 29 ó 30 días alternativamente. El novilunio anterior a la fecha dada fue ese año el día 8 de enero ${ }^{50}$; si le sumamos los 25 días señalados en la inscripción veremos que coincide exactamente con el 2 de febrero. No es una forma de indicar el día que haya podido constatar en otras inscripciones hispanas conocidas, lo cual concede un especial interés a ésta. Es un sistema que sí se utiliza de cuando en cuando en la epigrafía romana e incluso alguna inscripción cristiana lo emplea, aunque con mucha menor asiduidad.

\section{CONCLUSIONES}

En conjunto, se observa como esta inscripción procedente del monasterio de San Esteban de Bañolas refleja perfectamente las influencias culturales, políticas y sociales de su lugar de procedencia. Es un ejemplo que reivindica, si ello aún fuese necesario, la importancia de la Epigrafía y de la producción epigráfica como reflejo de una sociedad determinada. Los epígrafes son el espejo fiel de la sociedad que los produce, aunque teniendo muy en cuenta que es un instrumento de difusión publicitaria de unas ideas que está al servicio fundamentalmente de las clases dominantes, especialmente la iglesia, que es donde reside la cultura de la época. En este sentido se convierten en un elemento más para el conocimiento de las comunidades monásticas de aquellos tiempos.

En este caso concreto la inscripción de Bañolas nos pone en contacto con una sociedad, la de la Marca Hispánica, que es un crisol de influencias.

\footnotetext{
47 P. DE MARCA, Marca Hispanica sive limes hispanicus, Paris, 1688, app. CXII, col. 900.

48 F. UDINA MaRtorell, El Archivo condal de Barcelona. Estudio crítico de sus fondos, Barcelona, 1951, p. 52.

49 J.M. FONT I Rlus, Cartas de población y franquicia de Cataluña, Madrid-Barcelona, 1969, p. 18 , doc. 9.

so Ver J. Agusti, P. Voltes y J. Vives, Manual de cronología española y universal, Madrid, 1952, pp. 21-22, 25 y 185.
} 
Por un lado el poso visigodo y propiamente hispano que aún se mantiene, observable por ejemplo en la datación por la era. Por otro, los influjos procedentes del norte de los Pirineos, resultado del dominio político carolingio sobre los condados catalanes que se traslada al mundo de la religión y la organización eclesiástica. Eso encuentra eco en los aspectos externos, con una inscripción carente casi de decoración, una escritura que puede ser catalogada de carolina, cuando este tipo de letra no va a ser empleada en el resto de la Península hasta bastante tiempo después, y una forma de data que junto a la era hispánica utiliza otros sistemas que la vinculan con Europa, el año de Cristo y la indicción.

Por último, constatar la importancia en la investigación epigráfica de tener siempre en cuenta a quien corresponde la autoría moral de la inscripción, es decir quien encarga su ejecución. En este caso, probablemente se trate de la comunidad monástica de Bañolas, monasterio benedictino y por ello vinculado a la reforma cluniacense. La importancia que en Cluny se da a la liturgia funeraria fomenta el grabado de inscripciones como la aquí estudiada, a diferencia, por ejemplo, de lo que sucede en los monasterios cistercienses, donde se tienden a eliminar de sus claustros las inscripciones necrológicas ${ }^{51}$. Las diferencias litúrgicas existentes entre ambas Órdenes quedan así perfectamente patentizadas en su muy diferente producción epigráfica. Es éste un elemento que muestra de modo fehaciente como la producción epigráfica refleja el contexto religioso y cultural en el que se produce y al mismo tiempo se convierte en objeto que permite una mejor reconstrucción del modo de vida de aquellos monjes, sus intereses, su religiosidad y, en suma, su historia.

51 GARCíA LOBO, «De epigrafía cisterciense. Las inscripciones del monasterio de Carracedo", Cistercium, 208 (1997), p. 191. MARTiN LÓPEZ, “De epigrafía cisterciense: las inscripciones del monasterio de San Andrés de Arroyo", Cistercium, 208 (1998), p. 491. 